\title{
Health literacy of aging community in the northeastern Iran
}

\begin{abstract}
Introduction: In recent years, many studies have reported on the relationship between health literacy and health outcomes such as health status, chronic disease and hospitalization. Considering the increasing number of elderly people, studies need to be done on how to providing services to the elderly, also on recognizing the needs and health indicators of this age group. Therefore, the present study addresses the health literacy index of the elderly in the elderly population of northeastern Iran.
\end{abstract}

Method: This is a cross-sectional descriptive-analytic study carried out in 2019. About 190 elderly living in the northeastern Iran were selected by multi-stage (cluster and stratified) sampling method. Data were collected using a two-part questionnaire (demographic information- adult health literacy questionnaire) and analyzed by SPSS-20 software.

Results: Most of the elderly studied had an inadequate level of health literacy $(40.5 \%)$ $23.3 \%$ of the elderly had a borderline health literacy level. Only $36.3 \%$ of the elderly had adequate health literacy.

Conclusion: The results of this study showed that the elderly studied are lacking adequate health literacy, which is a serious challenge for health care providers and all providers of health services due to the growing number of elderly population in the country.

Keywords: elderly, health literacy, functional health literacy in adults
Volume 4 Issue 5 - 2019

\author{
Seyed Hamid Hosseini,' Rezvan Rajabzadeh, ${ }^{2}$ \\ Abdolrahim Asadollahi, ${ }^{3}$ Maryam Rabiey \\ Faradonbeh, ${ }^{4}$ Nasim Pirzadeh ${ }^{4}$ \\ 'PhD Candidate for Health Promotion, School of Health, Tehran \\ University of Medical Sciences, Iran \\ ${ }^{2} \mathrm{PhD}$ Candidate for Epidemiology, Department of Epidemiology, \\ Student Research Committee, Iran University of Medical \\ Sciences, Iran \\ ${ }^{3} \mathrm{PhD}$ in Gerontology, Department of Aging Health, Shiraz \\ University of Medical Sciences, Iran \\ ${ }^{4} \mathrm{MSc}$ in Aging Health, Department of Aging Health, Shiraz \\ University of Medical Sciences, Iran
}

Correspondence: Nasim Pirzadeh, MSc in Aging Health Department of Aging Health, School of Health, Shiraz University of Medical Sciences, 3rd Floor, Razi Ave., Shiraz, Iran, Email N.pirzadeh7238@gmail.com

Received: May II, 2019 | Published: September 12, 2019

\section{Introduction}

Health literacy consists of cognitive and social skills, which determine the motivation and ability of individuals to access, understand and use information in a way that maintains and improves their health. ${ }^{1}$ Also, health literacy includes a set of reading, listening, analyzing, decision-making skills and ability to apply these skills in health situations that necessarily does not relate to years of education or the general ability to read. ${ }^{2}$ Although it is still unclear to what extent health literacy affects health outcomes, many reasons indicate that many unpleasant health consequences are the results of inadequate literacy. ${ }^{3}$ Health literacy is now introduced as a global issue and debate. ${ }^{4,5}$ Low levels of health literacy are associated with poorer health outcomes and less use of health services. ${ }^{6}$ In recent years, many studies have reported on the relationship between health literacy and health outcomes, including health status, chronic disease and hospitalization. ${ }^{7}$ The development of primary health cares, improvement in economic and social conditions, and the emergence of new technologies for prevention, diagnosis, and treatment of diseases have led to increase in the population of people aged 60 and older, which are called elderly, according to the WHO's definition. ${ }^{8}$ Based on census of the Statistical Centre of Iran and studies performed, the population aged over 60 years in Iran in 2021 is estimated to be more than $10 \%$ of all population. ${ }^{9}$ This trend shows that studies should focus on how to deliver eldercare services, also recognizing the needs and health indicators of this age group. ${ }^{10}$ This study was performed to measure the health literacy index in the elderly population in the northeastern Iran.

\section{Method}

This is a cross-sectional descriptive-analytic study done in 2019 .
The statistical population of this research was elderly (over 60 years old) living in the northeastern Iran. Using previous studies, ${ }^{11}$ the sample size for the research was estimated to be 190 person. Sampling was done by multi-stage (cluster and stratified) method. Out of 29 bases and health centers in Bojnurd, 10 bases were randomly selected and among the elderly covered by each base, 20 person (over 60 years old) were selected almost equally in terms of gender variable. The inclusion criteria were to have voluntary consent and at least 60 years of age. The exclusion criteria were the lack of willingness to complete and fill the questionnaires and some degree of cognitive impairment. The questionnaire was filled out using face to face interview method. Before completing the questionnaires, In addition to explaining the purpose of the research project implementation for the individuals and informing them that their participation in the study is optional and notifying them of the confidentiality of the information collected, oral consent was obtained from the volunteers. A two-part questionnaire including demographic information - Test of Functional Health Literacy in Adults (TOFHA) was used to collect data. The validity of Persian version of The Adult Functional Health Literacy Questionnaire (TOFHA) was verified by Benihasem Tehrani at al. ${ }^{11}$ In the article by Javadzadeh et al. it is mentioned that this questionnaire was validated by Raeesi at al. for the elderly in Isfahan, which has a reliability of 0.79 for the computing section and 0.88 for the reading section (3) and has 2 computational and reading comprehension sections; reading comprehension section examines the patient's ability to read real texts related to health care, which includes 50 questions, and the computing section includes a series of descriptions on prescribed medications, visiting time, steps of getting financial assistance and an example of the outcome of one medical test. After handing over these descriptions in the form of cards to each person, they were asked the relevant questions (17 questions), and for the elderly who did not 
have the reading and writing literacy, the cards were being read by the questioner. Descriptive statistics (mean, standard deviation, frequency percentage) and analytical statistics (independent t-test, one-way analysis of variance and Tukey's post hoc test and linear regression) and SPSS version 25 (IBM Co.) were used to analyze the data.

\section{Results}

In this study, the age range of individuals was between $60-85$ years and the mean age of them was $66.3 \pm 5.67$ years. In terms of gender, $60 \%$ were female and $40 \%$ were male, and most participants were married $(88.9 \%)$. In terms of education, $48.4 \%$ of the elderly studied had primary and secondary education and $36.8 \%$ were illiterate, and only $2.1 \%$ had a university degree. The highest percentage of jobs was for housekeeping $(50.5 \%)$ and the lowest was for state employees (4.2\%). $60.5 \%$ of the elderly were head of household. The average number of people living with the elderly in a household was 2.87 and the standard deviation was 1.54 . Most of the elderly studied had a history of hospitalization (56.3\%). Regarding the level of health literacy among the elderly studied, the results showed that most of the elderly had insufficient health literacy level (40.5\%). $23.3 \%$ of the elderly had a borderline health literacy level. And only $36.3 \%$ of the elderly had adequate health literacy. Based on independent t-test, the results showed that the level of men literacy was significantly higher than that of women $(\mathrm{P}-$ value $=0.013)$. The results of the variance analysis showed that there was a statistically significant difference between the health literacy levels of the elderly with different levels of education (P-value $<0.001$ ). Tukey's post hoc test showed that this difference between the illiterate elderly and the rest of the groups was significant except for university education. Insignificancy between the illiterate group and the group with university education can be due to the low sample size in the group with university education. The results show that people with a history of illness had significantly higher levels of health literacy (P-value $=0.022$ ). The results of linear regression showed that with the control of the education level and the history of hospitalization, the men health literacy rate is 0.153 more than women. In addition, with the control of gender and hospitalization, the level of health literacy increases one rank to 0.26 . The mean and median health literacy score aligned from 100 elderly people was 3.95 and 66.06, respectively, and the standard deviation was 23.48. The mean and median health literacy score aligned from 50 elderly in the reading comprehension section was 28.64 and 29.55, respectively, with a standard deviation of 14.50 , and in the computing literacy section, the mean and median score aligned from 50 elderly were 35.32 and 40, respectively, and standard deviation was 13.21. Furthermore, the findings showed that there was no statistically significant relationship between age and health literacy of the elderly studied $(\mathrm{P}=0.245)$. The results of the Analysis of variance showed that there was no statistically significant difference in the health literacy of the elderly studied based on the occupation.

\section{Discussion and conclusion}

The results of this study indicate low health literacy among elderly living in Bojnurd in North Khorasan. Regarding health literacy score, most of the elderly studied had inadequate and borderline health literacy (63.8\%) and only $36.3 \%$ of them had adequate health literacy. The study by Izadi Rad at al Indicates that only $32 \%$ of the participants in the study had sufficient health literacy. ${ }^{2}$ Furthermore, in the study of Tavousi et al, it has been mentioned that $44 \%$ of participants have a limited health literacy level. ${ }^{12}$ In the study of Mohaddes Hakkak et al on pregnant women referred to health centers in Bojnurd, results showed that $61.9 \%$ of people studied had a great level of health literacy. ${ }^{13}$ The results of this study showed that there is a statistically significant difference between the health literacy level of the elderly studied with different level of education. This result is in the same direction with the study of Izadi Rad et al, Raeesi et al, also Tavousi at al. ${ }^{2,12,14}$ The study of Sandy Carollo, which reports on low literacy in elderly women, indicates that people with low education have lower levels of health literacy ${ }^{1}$ which is consistent with the results of our study. Tukey's post hoc test showed that the difference between the illiterate elderly and the rest of the groups was significant except for university education. Insignificancy between the illiterate group and the group with university education can be due to the low sample size in the group with university education. The results of the present study indicate that there is no statistically significant relationship between age and health literacy of the elderly studied. While there is a statistically significant relationship between age and health literacy in the study of Tavousi et al and Sahrayi et al, so that people with a higher age level have lower levels of health literacy, ${ }^{4,12}$ in this study a statistically significant relationship between gender of elderly and health literacy was observed, so that The level of men literacy was significantly higher than that of women, which was consistent with the results of the study by Borji et al, ${ }^{15}$ Fouladi et al, ${ }^{16}$ and Saatchi et al. ${ }^{15-17}$ The results show that people with a history of illness had significantly higher health literacy, so that the health literacy level of those with a history of hospitalization was 0.164 higher than those who did not have a history of hospitalization. In this regard, in the study of Cho Yang, it has been mentioned that improving health literacy can be an effective strategy for improving health status and reducing the use of hospital services and emergency services among the elderly. ${ }^{7}$ In this study, completing the questionnaire was difficult due to the large number of questions for the elderly, especially the elderly who did not have the literacy to read and write. On the other hand, most of the tools for assessing health literacy and evaluating physical health have not been culturally localized. It is suggested to translate each of these tools into native languages of different parts of Iran and use them because many elderly in different regions of Iran do not have complete mastery of the Persian language, or it is suggested to use native questioners to obtain accurate information from the elderly during the research.

\section{Ethical considerations}

Ethical matters e.g. plagiarism, informed consent, misconduct, data fabrication and/or falsification, double publication and/or submission, redundancy, etc. have been totally observed by the authors.

\section{Authors' contributions}

SHH \& RR contributed to the design, performed the interviews, analyzed and interpreted the interviews. MR\&NP have written the draft. AA have contributed discussion, also, revised the content, scientific writing, and approved the final manuscript as well.

\section{Funding}

$$
\text { None. }
$$

\section{Acknowledgements}

The authors express their appreciation to the anonymous referees for their advantageous comments and also declare that there is no conflict of interests as well. 


\section{Conflicts of interest}

The authors declare no conflict of interest.

\section{References}

1. Carollo S. Low health literacy in older women: the influence of patientclinician relationships. Geriatric nursing (New York, NY). 2015;36(2 Suppl):S38-42.

2. Izadirad H, Zareban I. The relationship of health literacy with health status, preventive behaviors and health services utilization in baluchistan, Iran Journal of Education and Community Health. 2015;2(3):43-50.

3. Javadzade SH, Sharifirad G, Radjati F, et al. Relationship between health literacy, health status, and healthy behaviors among older adults in Isfahan, Iran. J Educ Health Promot. 2012;1:31.

4. Sahrayi M, Panahi R, Kazemi S, et al. The study of health literacy of adults in karaj. Journal of Health Literacy. 2017;1(4):230-238.

5. Nutbeam D, Kickbusch I. Advancing health literacy: a global challenge for the 21st century. Health Promotion International. 2000;15(3):183-184.

6. Berkman ND, Sheridan SL, Donahue KE, et al. Low health literacy and health outcomes: an updated systematic review. Annals of internal medicine. 2011;155(2):97-107.

7. Cho YI, Lee SY, Arozullah AM, Crittenden KS. Effects of health literacy on health status and health service utilization amongst the elderly. Social science \& medicine. 2008;66(8):1809-1816.

8. Hosseininesar M, Zanjani H, Seyed Mirzaie SM, et al. Study of quality of life of the elders in gilan and its effective factors. Journal of Iranian Social Development Studies. 2018;10(1):75-88.
9. Mortazavi H, Pakniyat A, Ganji R, et al . The effect of self-management education program on disability of elderly patients with knee osteoarthritis referring to elderly care clinic of imam reza (AS) treatment center in shiraz, 2015-2106. Journal of North Khorasan University of Medical Sciences. 2017;8(3):461-470.

10. Asadi Noghabi A, Alhani F, Peyrovi H. The concept of health in elderly people: a literature review. Iran Journal of Nursing. 2012;25(78):62-71.

11. Mohseni M, Khanjani N, Iranpour A, et al. The relationship between health literacy and health status among elderly people in kerman. Salmand: Iranian Journal of Ageing. 2015;10(2):146-155.

12. Mahmoud Tavousi, AliasgharHaeri Mehrizi, shahram Rafiefar, et al. Health literacy in Iran: findings from a national study. Health Monitor Journal of the Iranian Institute for Health Sciences Research. 2016;15(1):95-102.

13. Hamidreza Mohaddes Hakak HJ, Rezvan Rajabzadeh, Davood Robatsarpooshi, et al. Health literacy level and related factors among pregnant women referring to bojnord health centers in 2017. International Journal of Pharmaceutical Research. 2019;11(1):152-158.

14. Reisi M, Mostafavi F, Hasanzadeh A, et al. The Relationship between health literacy, Health status and healthy behaviors among elderly in Isfahan. 2011..

15. Borji M, Tarjoman A, Otaghi M, et al. Health literacy level and its related factors among the elderlies in ilam in 2015. Iran Journal of Nursing. 2017;30(108):33-43.

16. Fouladi N, Hazrati S, Shabani M, et al. Investigating middle-aged health literacy in ardabil. Journal of Health Literacy. 2017;2(1):39-44.

17. Saatchi M, Panahi M, Ashraf Mozafari A, et al. Health literacy and its associated factors: a population-based study, hormuz Island. Iranian Journal of Epidemiology. 2017;13(2):136-44. 